\title{
First Description of Tricuspid Valve Dysplasia in a Basset Hound
}

\author{
Iuliu SCURTU* \\ Internal Medicine Department, Faculty of Veterinary Medicine. University of Agricultural Sciences eand \\ Veterinary Medicine, Mănăștur street 3 - 5, Cluj-Napoca, Romania \\ *corresponding author: iuliu.scurtu@usamvcluj.ro
}

Bulletin UASVM Veterinary Medicine 75(1)/2018

Print ISSN 1843-5270; Electronic ISSN 1843-5378

doi:10.15835/buasvmcn-vm:004117

\begin{abstract}
This report represents the first description of tricuspid valve dysplasia in a Basset hound. We evaluated clinical signs, ECG abnormalities and echocardiographic findings related to tricuspid valve dysplasia in a 6 month Basset Hound. A specific protocol of cardiologic examination was performed every time the patient was evaluated. This protocol included: clinical exam, ECG and echocardiographic investigation. The clinical examination identified over the entire period of surveillance a dog with good body condition score (2.5-3/5), alert, bright, being able to take moderate exercise. A 4/6 left heart base systolic murmur was identified at every examination. ECG revealed a sinus rhythm. Echocardiography identified right heart abnormality, with malposition of tricuspid annulus, one leaflet being short and thick and other extremely long with abnormal motion. Due to abnormal morphology of tricuspid valve apparatus a tricuspid regurgitant jet was noted with Color Doppler. The left heart seemed to have normal morphology. An atrial septal defect was also noticed. Further studies need be performed in order to establish if tricuspid valve dysplasia in Basset hound is a congenital or genetic defect.
\end{abstract}

Keywords: Basset hound, congenital heart defect, echocardiography, tricuspid dysplasia

\section{Introduction}

Tricuspid valve dysplasia (TVD) represents $3.1 \%$ of dog heart congenital defects (Oliveira et al., 2011). In another study, TVD represents $2 \%$ of canine congenital heart disease (Schrope, 2015). This defect evolves mainly in large breed dogs, Golden Retrievers being overrepresented, but this defect also develops in other breeds like: Dogue de Bordeaux (Ohad et al., 2013), German shepherd, Great Dane, Old English Sheep dog or Weimaraner. It is one report of TVD in the tsushima leopard cat (Shimamura et al., 2017). TVD, also called Ebstein' anomaly in humans, is characterized by different features: adherence of the tricuspid leaflets to the myocardium, downward displacement of the tricuspid annulus, dilation of the uppermost part of the right ventricle, redundancy and fenestrations of the anterior leaflet of tricuspid valve and dilation of the tricuspid annulus (Dearani et al.,
2003; Yuan et al., 2003; Andelfinger et al., 2003). Usually, the septal leaflet is displaced toward the apex and the anterior leaflet, the biggest in normal condition has a "sail like" appearance and is correctly positioned (Hetzer et al., 1998).

\section{Material and methods}

Clinical examination consisting of specific cardiac examination was focused on heart auscultation, palpation of the thorax for precordial trill identification, evaluation of the jugular and peripheral veins and evaluation of the pulse characteristics.

Echocardiographic examinations implied two dimensional (2D), M-mode and Doppler evaluations of the heart structures. Echocardiographic exam was performed with an Esaote MyLab 40 Vet device equipped with a phased array, multifrequency transducer (4-7.5 MHz). The hair was 

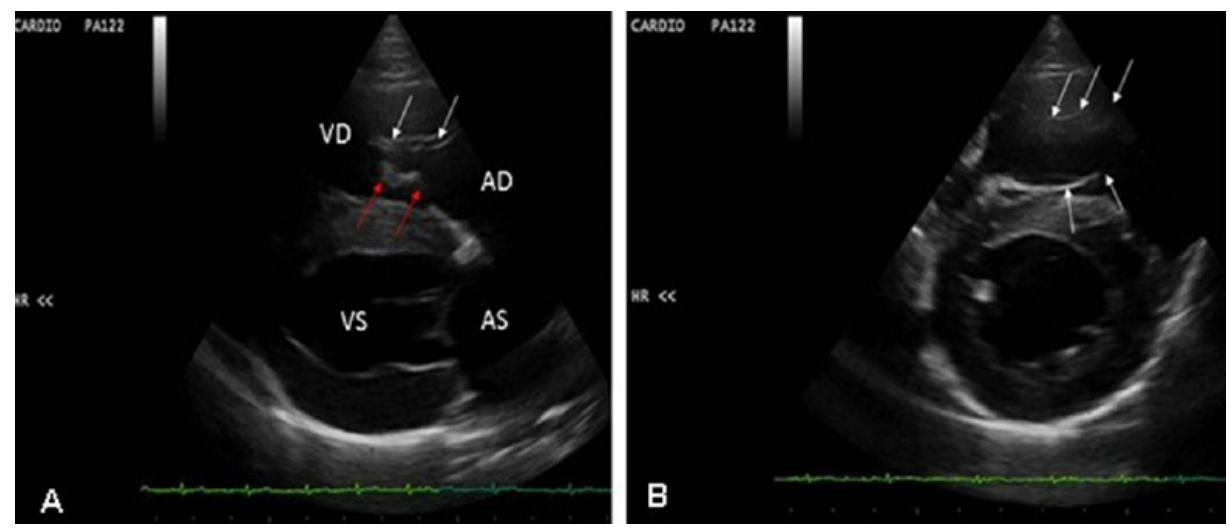

Figure 1. A. Long axis, four chamber view. Malposition of the tricuspid valve. B. Cross section at papillary muscle level. VD - right ventricle; VS - left ventricle; AS - left atrium; AD - right atrium; white arrow - anterior leaflet of the tricuspid valve; red arrow - septal leaflet of the tricuspid valve

clipped from the right and left sides and echocardiographic examination was performed using a cut-out table from underneath. We used right side, four chamber long axis view for general evaluation of the heart structures (the ratio between chambers, the ratio between walls and position of valve leaflets). Left ventricle measurements were performed in M-mode, from short axis view, at the papillary muscle level. Five consecutive measurements were evaluated and an average was calculated. Pulmonary flow was evaluated from the right side, at the level of the heart base and aortic flow was investigated from the left side, apical view. Doppler examination of the atrioventricular valves was done from apical four chamber view, the volume sample being placed at the tip of the mitral and tricuspid leaflets.

An electrocardiogram (ECG) was recorded with a digital device (PolySpectrum) using 6 standard leads. For this procedure the dog was gently restrained in right lateral recumbency and the examination was performed over a 5 minutes period.

Systolic and diastolic tensions were recorded by a non-invasive technique (high definition oscillometry).

\section{Results and discussions}

A 6 month old, privately owned, Basset hound male, $15.2 \mathrm{~kg}$ was evaluated at the Cardiology Department of the Veterinary Medicine Faculty, Cluj-Napoca. The owner did not report any abnormality with the dog before examination, this exam being a standard procedure for the breeder before selling. The dog had a good body condition score (2.5/5). Mucous membranes were moist and light-pink. No jugular or peripheral veins abnormality was noted. No ascites and no peripheral edema were identified. The pulse was normal. Heart auscultation identified on each hemithorax a systolic murmur with peak of maximum intensity over the right heart base $(4 / 6)$. On the left hemithorax the murmur was graded as $3 / 6$. No precordial trill was identified.

The ECG exam identified sinus arrhythmia. Echocardiographic examination revealed a heart with abnormal morphology. The right atrium was extremely dilated. Tricuspid annulus was malpositioned, being displaced toward the apex of the heart.

Tricuspid valve leaflets had abnormal structure; the septal leaflet was short and thick and presented short chordae tendineae and the anterior leaflet was longer than expected (Fig.1). Color flow Doppler evaluation of the tricuspid valve identified a turbulent flow across the valve.

This jet had $3.71 \mathrm{~m} / \mathrm{s}$ peak velocity with abnormal profile (V shape) (Fig.2). The left heart had normal morphology. No abnormal jet was identified across the mitral valve. Aortic flow had an asymmetric configuration, with a peak velocity around $1 \mathrm{~m} / \mathrm{s}$. Pulmonary flow had a symmetric shape and normal velocity. The interatrial septum had abnormal morphology. It seemed to be formed of two membranes which were not completely attached over the entire surface of the septum. We suspected also the presence of an atrial septal defect, but we did not identify any jet across the interatrial septum. 


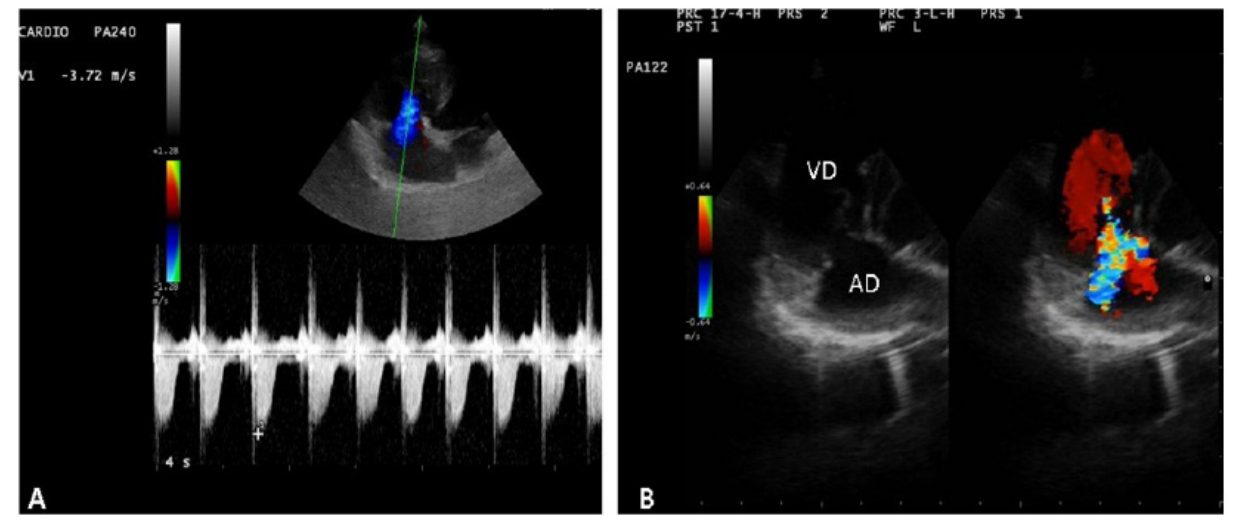

Figure 2. A. Continous wave Doppler. The presence of a tricuspid regurgitant jet with $3.72 \mathrm{~m} / \mathrm{s}$ peak velocity. B. Dual mode (2D and Color flow Doppler). A tricuspid regurgitant jet. VD - right ventricle; AD - right atrium.

Both, systolic and diastolic blood pressure was within reference range limits.

This report represents the first description of the TVD in a Basset Hound dog. Clinical exam identified a systolic right heart base murmur, which is not a pathognomonic finding for TVD. Moreover, at the time of examination, the dog was doing fine and the owner did not mention any abnormality. More likely, in case of Labrador Retriever or Golden Retriever, a right heart base systolic murmur might raise the suspicion of tricuspid dysplasia, because these breeds are predisposed, but in the Basset Hound TVD would be at the bottom of the differential diagnosis list. Clinical signs associated with TVD are: exercise intolerance, abdominal distension and respiratory distress (Chetboul et al., 2004). Pulmonary hypertension is a possible complication of TVD, as a result of right ventricle dilation and increased blood flow into pulmonary vasculature worsen tricuspid regurgitant jet significantly. At the same time pulmonary hypertension exacerbates the right-side heart failure (Choi et al., 2009).

In our case the ECG was absolutely normal, while in another reports a splintered QRS complex is a common finding (Kornereich et al., 1997). Kornereich found splintered QRS complexes in 9 of 19 Labrador Retrievers and 12 of 20 nonLabrador Retriever dogs with tricuspid dysplasia.

Other ECG abnormalities identified in case of tricuspid valve dysplasia were atrial fibrillation or supraventricular tachycardia (Kornereich et al., 1997) or giant $P$ waves (Choi et al., 2009). The cause of splintered QRS complexes has not been identified yet, but potential causes might be represented by fibrosis, bypass tracts and ischemia (Kornreich et al., 1997).

The gold standard method for the TVD diagnosis is echocardiographic examination. In our case, the septal leaflet was short, thick and with downward displacement, while anterior leaflet was positioned in the right place but had "sail like" appearance. Because of the abnormal tricuspid apparatus due to abnormal leaflets, a tricuspid regurgitant jet was present. Tricuspid valve dysplasia could evolve as an isolated pathology or in combination with other congenital diseases such as atrial septal defect, mitral valve dysplasia or pulmonic stenosis (Famula et al. 2002). We suspect that tricuspid dysplasia in our report evolves in combination with an atrial septal defect. Abnormal morphology of the interatrial septum raised this suspicion, but we did not identified any flow between atria. Tricuspid regurgitation might increase right atrium pressure, so the pressure in left and right atrium might be similar, so no jet has been present between these chambers at the time of examinations. Probably, as the disease will evolve, a continuous increase of the pressure in the right atrium will give us the chance to identify an abnormal flow from right to left. Two weeks after first examination, the owner claimed that the dog got cyanotic after a short burst of run with another dog, which could be evidence for a possible shunt between right and left heart.

In humans, TVD also called Ebstein's anomaly, represents an embryologic defect (Sherwin et al., 2017). New evidence has suggested that this congenital defect in humans may be related to the mutation in the myosin heavy chain (Yuan et al., 2017). In a purebred Labrador Retriever 
with TVD, a possible anomaly in chromosome 9 was suspected (Andelfinger et al., 2003), while in Dogue de Bordeaux it was proposed an autosomal recessive mode of inheritance (Ohad et al., 2013).

In humans, treatment implies surgical reconstruction of the tricuspid valve (Huang et al., 2017), a procedure that has not been routinely implemented in veterinary medicine. In veterinary medicine, treatment options include administration of oxygen, diuretics, positive inotropic drugs, abdominocentesis or treatment of pulmonary hypertension.

\section{Conclusions}

This report describes for the first time TVD in a Basset Hound. This dog being a purebred and having complete information about its pedigree, might be the starting point for the study of this heart defect in Basset Hound, to establish if this defect has a genetic determinism.

Acknowledgments. This research did not receive any specific grant from funding agencies in the public, commercial, or not-for-profit sectors.

\section{References}

1. Andelfinger G, Wright KN, Lee H, Siemens L, Benson D (2003). Canine tricuspid valve malformation, a model of human Ebstein anomaly, maps to dog chromosome 9. J Med Genet, 40: 320-324.

2. Chetboul V, Tran D, Carlos C, Tessier D, Pouchelon JL (2004). Congenital malformations of the tricuspid valve in domestic carnivores: A retrospective study of 50 cases. Schweiz Arch Tierheilkd, 146: 265-275.

3. Choi R, Lee KS, Moon HS, Park IC, Hyun C (2009). Ebstein's anomaly with an atrial septal defect in a jindo dog. Can Vet J, 50: 405-410.
4. Dearani JA, Danielson GK (2003). Tricuspid valve repair for Ebstein's anomaly. Operat Tech Thor Card Surg, 8: 188192.

5. Famula TR, Siemens LM, Davidson AP, Packard M (2002). Evaluation of the genetic basis of tricuspid valve dysplasia in Labrador Retrievers. Am J Vet Res, 63: 816-820.

6. Hetzer R, Nagdyman N, Ewert P, Weng YG, Meskhisvili VA, Berger F, Pasic M, Lange PE, (1998). J Thorac cardiovasc Surg, 115: 857-868.

7. Huang SC, Wu ET, Chen SJ, Shih JC, Chou HW, Chang CI, Chiu IS, Chen YS (2017). Surgical strategy toward biventricular repair for severe Ebstein anomaly in neonates and infancy. Ann Thorac Surg, S0003 - 4975: 30195-30199.

8. Kornreich BG, Moise NS (1997). Rightatrioventricularvalve malformation in dogs and cats: an electrocardiographic survey with emphasis on splintered QRS complexes. J Vet Intern Med, 11: 226-230

9. Ohad DG, Avrahami A, Waner T, David L (2013). The occurrence and suspected mode of inheritance of congenital subaortic stenosis and tricuspid valve dysplasia in Dogue de Bordeaux dogs. The Vet Journal, 197: 351-357.

10. Oliveira P, Domenech O, Silva J, Vannini S, Bussadori R, Bussadori C (2011). Retrospective review of congenital heart disease in 976 dogs. J Vet Int Med, 25: 477-4783.

11. Schrope DP (2015). Prevalence of congenital heart disease in 76.301 mixed - breed dogs and 57.025 mixed - breed cats. J Vet Card, 17: 192-202.

12. Sherwin ED, Abrams DJ (2017). Ebstein anomaly, Card Electrophysiol Clin, 9: 245-254.

13. Shimamura S, Shiota Y, Takagi N, Nishimura S, Tani H, Shimada T (2017). Ebstein anomaly in the tsushima leopard cat, J zoo Wild Med, 48: 585-589.

14. Yuan SH (2017). Ebstein's anomaly: genetics, clinical manifestations and management, Pediatrics and Neonatology, 58: $211-215$. 